\title{
A detailed analysis of the lunar and Phobos nodes within the OASIS spaceports network
}

\author{
Lucie Poulet ${ }^{1}$ \\ DLR Institute of Space Systems, Bremen, Germany, 28359 \\ Marc Labriet ${ }^{2}$ \\ International Space University, Illkirch, France, 67400 \\ and \\ Chrishma Singh Derewa ${ }^{3}$ \\ International Space University, Illkirch, France, 67400
}

\begin{abstract}
A network of spaceports with specific waypoint locations combining In Situ derived propellant generation with consumables such as water and oxygen provides a sustainable vision of solar system industrialization and enables the development of long term space exploration. The Operations And Service Infrastructure for Space (OASIS) network architecture was designed to facilitate affordable access to orbital and deep space destinations. It consists of three nodes located in Low Earth Orbit (LEO), the Moon and finally Mars' moon, Phobos. Node 2 is based in the Lunar South Pole, where trapped water in craters, almost constant illumination on the craters' rim, and small temperature gradients offer one of the best environment. Initially unmanned, the lunar outpost is composed of a spaceport to land and launch vehicles safely, a power plant, and an in-situ resources processing plant. Once extracted from the lunar regolith, water is separated into hydrogen and oxygen which can be used as propellant for various spacecraft. It is then sent to LEO to make inexpensive fuel accessible from Earth and is used on site to support habitation, human operations and lunar launches. Node 1 will also refuel the CARAVAN (Cargo Autonomous Rendezvous and Velocity Adjustment/Navigation) orbital tug capable of intercepting and accelerating missions to more distant destinations. The Martian moon, Phobos, is the final Spaceport node in the OASIS architecture. Missions to the red planet will refuel, repair and prepare on Phobos prior to descent on Mars and before returning to Earth or going onwards. Propellant generation and storage facilities, as on node 1, allow node 3 to provide resources mined from wet asteroids, such as Ceres, the moons themselves or raised from the Martian ice caps. Meeting the technical challenge of extending the presence of humans throughout the solar system, this network will enable shorter delivery times, reduce space exploration and development costs, and increase mission mass to the Martian surface.
\end{abstract}

$\begin{array}{ll}\text { CARAVAN } & =\text { Nargo Autonomous Rendezvous and Velocity Adjustment/Navigation } \\ \text { ESA } & =\text { European Space Agency } \\ \text { GEO } & =\text { Geostationnary Orbit } \\ \text { GTO } & =\text { Geostationary Tansfer Orbit } \\ \text { ISRU } & =\text { In Situ Resources Utilization } \\ \text { ISS } & =\text { International Space Station }\end{array}$

\footnotetext{
${ }^{1}$ Research Associate, System Analysis Space Segment, Robert-Hooke Str. 728359 Bremen, Germany.

${ }^{2}$ Project Manager, Advanced Services, 150 W Tasman Dr, San Jose, CA 95134.

${ }^{3}$ Master student, ISU Master, 67400 Illkirch, France.
} 


$\begin{array}{ll}\text { ISU } & =\text { International Space University } \\ K S C & =\text { Kennedy Space Center } \\ L E O & =\text { Low Earth Orbit } \\ L H_{2} & =\text { Liquid Hydrogen } \\ \mathrm{LO}_{2} & =\text { Liquid Oxygen } \\ M A V E N & =\text { Mars Atmosphere and Volatile EvolutioN } \\ \text { NASA } & =\text { National Aeronautics \& Space Administration } \\ \text { OASIS } & =\text { Operations And Service Infrastructure for Space } \\ R E S O L V E & =\text { Regolith and Environment Science and Oxygen and Lunar Volatiles Extraction } \\ S S P & =\text { Space Studies Program } \\ V T V L & =\text { Vertical Takeoff Vertical Landing }\end{array}$

\section{Introduction}

$\mathrm{T}$ HE cost of launching sufficient mass for long-duration missions towards deep space destinations is prohibitive. All consumables for any mission are typically carried with the spacecraft from the Earth surface. Consequently, with current launch systems, too much of the spacecraft's mass and volume is occupied by propellant, placing a great mass penalty on the mission and severely limiting the launch vehicle payload.

The OASIS (Operations And Service Infrastructure for Space) concept proposes a solution to this problem in the form of an in-space multi-purpose logistics network to provide overall support for exploration and commercial activities. The concept consists of placing propellant and other support items in a network of spaceports strategically located in the solar system that would be replenished, where possible, by In-Situ Resource Utilization (ISRU). The spaceports in this network would be composed of infrastructure waypoints providing services for spacecraft and facilitating their departure and arrival. The OASIS team has developed a phased plan spanning 50 years that proposes the step by step construction of the different nodes of this network, limiting the initial financial risks and strong political challenges by investing the commercial profits of each node into the next ${ }^{1,2}$.

Orbital fuel depots and lunar settlements have already been extensively studied. The novelty of the OASIS concept resides on the integration of the different nodes in a global network architecture. This end to end solution addresses a diverse set of mission objectives servicing not only the near-Earth market but deep space exploration objectives as well. An in-space logistics network will revolutionize how deep space travel is executed thus far. Cheaper propellants made from water at Spaceport Node 1 in Low Earth Orbit (LEO) will provide the revenue infrastructure to enable new services and, possibly, enhance or disrupt the space transportation market. Part of the long term plan will be to further develop Spaceport Node 2. This node will support human activities on the Moon, such as mining or tourism. It will allow launching heavier payloads to/or beyond the Moon. The flexibility of this architecture will support robotic missions to and beyond Mars and eventually facilitate human missions to Mars by sending significantly higher payload mass and/or reducing the flight time for humans. Lastly, access to the asteroid Ceres will enable a continuous supply of water to the red planet capable of not only supporting all the fuel requirements of missions to the Red Planet, but life support needs as well. This whole network will enable a regular and easy access to the inner solar system and will enable numerous exploration and science missions to the planets beyond the asteroid belt and to Keuper belt objects ${ }^{1,2}$.

\section{Description of OASIS network}

The spaceports network consists of four nodes: node 0 on Earth in Kennedy Space Center (KSC), node 1 in LEO, node 2 on the Moon, and node 3 on the Mars' moon Phobos. The OASIS network architecture is designed to support NASA's Global Exploration Roadmap and its ultimate destination: Mars, but also to allow exploration beyond the asteroid belt into the outer Solar System. This network of spaceports extends the current terrestrial architecture into space. Each node option was evaluated with the following criteria: accessibility, environment, costs, maturity of technology required, and most importantly contribution of each element to the network ${ }^{1,2}$.

The first node is an intermediate launch and staging location in LEO with propellant for refueling and primary subsystem services to be established during a first phase of the network development (2015-2025) (Fig. 1). The core service is the CARAVAN (Cargo Autonomous Rendezvous and Velocity Adjustment/Navigation) transportation service for GEO satellites. CARAVAN is a tele-operated spacecraft with robotic arms which transfers spacecraft from LEO to Geostationary Transfer Orbit (GTO) for orbital inclinations of $0^{\circ}$ to $51.6^{\circ}$ (ISS orbit) and circularizes their orbit to GEO, if necessary. Other services include on-orbit refueling at LEO, spacecraft repair, geostationary orbit slot change, de-orbit and salvage capabilities. By using this service, including eventual orbit inclination change, the client would be able to use a wider range of launch vehicles. It would not need to carry the upper stage 
and extra propellant needed to reach GEO. Preliminary analysis showed that Spaceport Node 1 would be commercially profitable and could reduce transportation costs to GTO up to $44 \%$ depending on the launch vehicle $e^{1,2}$.

Spaceport Node 2 node is placed on the Moon's surface in the next phase (2025-2045) (Fig. 1) and would first be a robotic base paving the way for future human presence. With almost continuous solar energy, an estimated 600 million tons of water ice at its north pole, and a resource-rich regolith, the Earth's Moon is the most promising and accessible location for in-situ production of propellants, solar panels, and habitation modules. The water excavated on the Moon's surface would be distributed across the network, especially at Spaceport Node 1, to produce cheaper propellants and lower the cost of space access.

Node 3 will be established on Phobos and start operations during a third phase from 2045 (Fig. 1) enabling missions to the Martian surface and beyond. Even though the presence of resources on Phobos have not been fully quantified and positioned, the reduced $\Delta \mathrm{v}$ required to reach resource-rich asteroids makes it a very attractive location for Spaceport Node 3.

The roadmap of the entire network is presented on Figure 1 and summarizes timeline of the three phases and node construction including missions, system elements set up, as well as critical technologies development ${ }^{1,2}$.

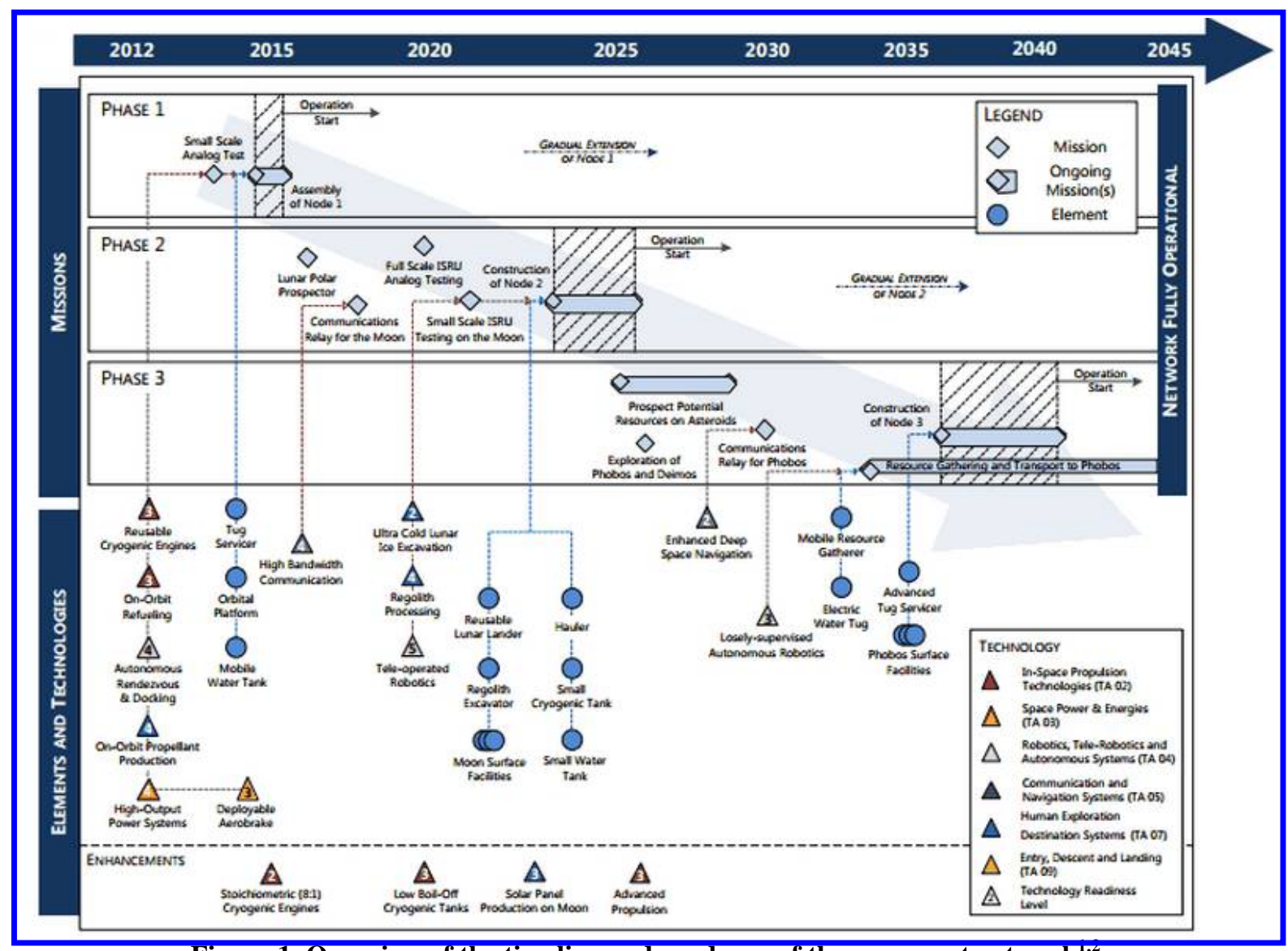

Figure 1. Overview of the timeline and roadmap of the spaceport network ${ }^{1,2}$.

\section{Node 2}

Spaceport Node 2 is a stepping stone to expand human presence into space and a crucial step for the development of Spaceport node 3 on Phobos. A system of elements, such as an excavator, an ISRU plant, a facility for propellant generation and water storage, a reusable Moon lander, as well as a spaceport to launch and land vehicles safely will be set up. Later on, when humans live on the base, consumables (oxygen, water, and food) will be produced using advanced life support systems. Operational support will be deployed in parallel, including power generation and communications systems.

American Institute of Aeronautics and Astronautics 


\section{A. Location of node 2}

The connecting ridge between the Sverdrup and Shackleton crater offers characteristics which would be suitable for the establishment of a Lunar base on the Moon:

- Permanently shadowed craters where temperature remains at about $40 \mathrm{~K}^{3}$ have shown large concentrations in hydrogen (data from Lunar Prospector in Feldman et al. 2001), which reveals a high probability of trapped water ice ${ }^{4}$.

- An average illumination duration of $89.01 \%$ and 3500 hours of uninterrupted sunlight ${ }^{5}$. Overall at the South Pole, a total of $2.25 \mathrm{~km}^{2}$ is illuminated $80 \%$ of the time in average ${ }^{5}$.

- Temperature gradients of only $20 \mathrm{~K}$ would allow equipment to function more efficiently. Indeed the minimum nighttime temperature is $160 \mathrm{~K}$ and the maximum daytime temperature is $180 \mathrm{~K}^{3}$.

\section{B. Preparing the node construction}

To prepare construction of node 2, the first mission will be a lunar polar prospector landed on the Moon surface, which will confirm the presence of trapped water in the South Pole craters, measure radiations doses at the surface and do a precise mapping of illumination as well as terrain inclination. Main elements of this mission will include a small laboratory and rovers. Rovers will explore the area in a diameter of several kilometers and collect samples to be analyzed by the local laboratory. They will be equipped with various probes enabling temperature, terrain inclination, radiation doses, and illumination measurements. Some rovers will be equipped with drilling capabilities, enabling to collect trapped water ice. This is already planned to be done by the NASA RESOLVE (Regolith and Environment Science and Oxygen and Lunar Volatiles Extraction) experiment to be launched in 2017/2018. The small laboratory will provide support to the rovers and analyze collected samples. Results of this mission will enable to determine a good location in the South Pole to establish the different elements of the lunar base: launch pad, ISRU processing plant, power plant, habitats.

About two years later, a mission will set up a high bandwidth communications relay for the Moon. This will ensure continuous communication from the Earth with the robots and rovers once they start the buildup of the base.

The third mission will be on Earth and be a full scale ISRU analog testing, involving all the equipment and technologies to be used on the Moon later on. This mission will enable to notice flaws in the processes and mechanisms of the isru plant and make adjustements accordingly to start developing space proofed technologies.

Finally a small-scale ISRU testing mission on the Moon, shortly after and taking into account the results of the full-scale terrestrial testing will confirm the lunar resources processing technologies. Especially ultra-cold lunar ice excavation, regolith processing and tele-operated robotics will need to be assessed.

\section{Unmanned lunar base}

Several missions will be needed to finalize the construction of the lunar base in the South Pole, which will start with utilizing teleoperated robots from Earth. In a first phase, large solar panels will be deployed on tall antennas above the lunar surface and will be linked to a charging station where rovers and robots will recharge their batteries when needed. Due to the long day/night cycles on the lunar surface, these rovers and robots will need to have to be autonomous in terms of power for a minimum of two days ${ }^{5}$. Excavation rovers will dig lunar regolith, until a sufficient large flat area is reached. Indeed a zero degree terrain inclination is desired to build a spaceport to launch and land vehicles safely. Other processing robots will transform the excavated regolith into very fine particles, making it ready for 3D printers utilization and build the ISRU processing plant. Although 3D printing is still an emerging technology, it is expanding very fast and has triggered interest of many space agencies, especially ESA which has recently shown a small prototype of a future lunar base made by 3D printers using lunar regolith ${ }^{6}$. The nuclear power plant will be built using the same method. Later on, replication technologies will be used to expand the lunar node.

Once the power plant and the ISRU processing plant are completed and operational, regolith processing can begin: regolith is used to build other lunar facilities and water is excavated using ultra cold lunar ice excavation and extraction technologies. It is then stored in water tanks waiting to be sent to spaceport node 1 by the reusable moon shuttle. $\mathrm{O}_{2}$ and $\mathrm{H}_{2}$ will also be extracted from the regolith and cooled to get cryogenic liquid propellants $\left(\mathrm{LO}_{2}\right.$ and $\mathrm{LH}_{2}$ ), which are needed to fuel spacecraft departing the Moon, and stored into a small cryogenic tank. It turns out that it requires much less energy to send water from the surface of the Moon than from the surface of the Earth, so the use of water from the surface of the Moon could greatly reduce the cost of propellants. Indeed there is enough water ice at the North Pole to sustain a shuttle flight every day for 2200 years (Indian lunar probe Chandrayaan-1). Such proof of concepts are currently being developed in numerous research centers, like the Kennedy Space 
Center's Swamp Works which is bulding a test bed for lunar operations, including a complete "dust to burst" simulation using regolith to power a rocket engine.

In the medium-term, producing propellant from lunar water would decrease the operating cost of Spaceport Node 1 by an estimated 38\% compared to the short-term solution of supplying water from Earth. Node 1 would also provide a transportation service from LEO to Lunar orbit. This service, coupled with the establishment of Spaceport Node 2, has the potential to greatly reduce the cost of future missions to the Moon, and enable the emergence of new markets related to human and robotic lunar operations.

\section{In situ resources processing plant}

ISRU plant processes excavated regolith to extract mainly water and oxygen and to refine regolith itself in order to build lunar facilities.

Permafrost and cold trap are the two main forms of water at the lunar poles. Cold trap water is found in ever dark craters $(40 \mathrm{~K})$ and is impossible to mine with current technologies. Permafrost water is found on the rim of polar craters and is mixed together with regolith below the surface, enabling an easier extraction using current technologies. After extraction of water, it can be stored in extracted-form or broken down into hydrogen and oxygen through electrolysis.

Current technologies for oxygen extraction include (Table 1$)^{1,2}$ :

- Electrolysis utilizing an acidic ionic liquid

- Extraction of water from permafrost utilizing microwaves

- Molten oxide electrolysis

Table 1. ISRU Processes for Oxygen Extraction from regolith.

\begin{tabular}{|c|c|c|c|c|c|}
\hline \multirow{2}{*}{$\begin{array}{l}\text { ISRU Process }\left(\mathrm{O}_{2} \text { from }\right. \\
\text { Regolith) }\end{array}$} & \multirow{2}{*}{$\begin{array}{l}\text { Regolith Excavation } \\
\text { Rate }(\mathbf{k g} / \mathbf{h})\end{array}$} & Reagent & \multirow{2}{*}{$\begin{array}{l}\text { Specific } \\
\text { Mass }\end{array}$} & \multirow{2}{*}{$\begin{array}{l}\text { Specific } \\
\text { Power }\end{array}$} & \multirow{2}{*}{ Efficiency } \\
\hline & & Output & & & \\
\hline \multirow{2}{*}{$\begin{array}{l}\text { Hydrogen reduction of } \\
\text { Ilmenite }\end{array}$} & \multirow{2}{*}{150} & $\mathrm{H}_{2}$ & \multirow{2}{*}{0.15} & \multirow{2}{*}{1.93} & \multirow{2}{*}{$1.41 \%$} \\
\hline & & $\mathrm{O}_{2}$ & & & \\
\hline \multirow{2}{*}{$\begin{array}{l}\text { Carbothermal reduction of } \\
\text { Silicates }\end{array}$} & \multirow{2}{*}{15} & $\mathrm{CH}_{4}$ or $\mathrm{CO}$ & \multirow{2}{*}{$\sim 0.1$} & \multirow{2}{*}{1.35} & \multirow{2}{*}{$\sim 14 \%$} \\
\hline & & $\mathrm{O}_{2}$ & & & \\
\hline \multirow{2}{*}{ Molten Silicates Electrolysis } & \multirow{2}{*}{10} & None & \multirow{2}{*}{0.065} & \multirow{2}{*}{1.5} & \multirow{2}{*}{$21.4 \%$} \\
\hline & & $\mathrm{O}_{2}$ & & & \\
\hline
\end{tabular}

\section{E. Spaceport to launch and land vehicles safely}

The launch and landing pad will be essential to lunar operations on the base to ferry excavated water back to LEO. It will be a Vertical Takeoff Vertical Landing (VTVL) pad that enables spacecraft to launch and land safely and accurately through the use of navigation beacons.

\section{F. Human presence}

Once the unmanned base is completed and functional, operations for preparing human presence will begin. This includes extracting $\mathrm{O} 2$ and water from lunar regolith and building human habitats. For radiation and meteorites protection purposes, human habitats will be underground or covered with a thick layer of lunar regolith. Advanced life support systems including recycling of organic wastes, food production through higher plants, and water and atmosphere management will be installed prior first human arrival. When the first astronauts arrive on the lunar base, they will be able to live into the habitat and further develop node 2. Further development includes building facilities for science experiments and testbed for critical technologies and bases for lunar tourism. It also includes 
preparing prospective missions for exploration of Mars crossing asteroids and asteroid belt, and rich water dwarf planets like Ceres to assess resources potential and prepare node 3.

\section{Node 3}

Phobos allows easier access to Mars with a delta-V from Mars surface to Phobos of $1.25 \mathrm{~km} / \mathrm{s}$ from an altitude of $200 \mathrm{~km}$ at the equator ${ }^{7}$ and enables to extend the spaceport network in the inner and outer solar system.

A satellite communication network around Mars allowing continuous communication with the Phobos base will be established prior node 3 construction, which will later on enable primary exploration of Mars with teleoperated robotic vehicles from Phobos. Indeed line of sight communications with Mars from Phobos is possible between latitudes of $\pm 64.8^{\circ}$ and for four hours every 11.1 hours $^{8}$. Communication with Polar Regions will be possible using orbiters such as Mars Atmosphere and Volatile EvolutioN (MAVEN) and ExoMars ${ }^{7}$. Operating robotic vehicles from orbit enables to get data in real time and thus make very quick decisions concerning experiments and exploration operations ${ }^{7}$.

Prospect missions on various asteroids will also prepare the builup of the Phobos base. Development of water mining on Ceres - largest asteroid in the belt and predicted to have more fresh water than Earth itself - with an accompanying distribution point at Phobos will drastically reduce mission costs to Mars, enable larger human crews and facilitate access to the outer planets. The CARAVAN transportation system will act as fuel tanker carrying water mined on Ceres back to Phobos using long duration electric propulsion. The development of a drilling and storage system on Ceres is a key component to the OASIS architecture that must be studied in details if this resource is to be exploited.

From potentially mining Phobos or other wet asteroids, it could become a port for transportation of resources and people throughout the Solar System in a third phase (2045 - onwards).

\section{Conclusion}

The conquest of space will not be achievable without a significant reduction in the effort and cost required for access. The OASIS concept for a network of spaceports extending existing transportation and logistics infrastructure on Earth into space has the potential to significantly reduce the cost of robotic and human space missions and to act as an enabler for more cost effective space exploration. The network described here is not exclusive to rare and expensive government programs, but can be shared with private enterprises on a large scale. This scalable initiative can be compared to the construction of roads and service stations in isolated regions on Earth. Only an infrastructure network using in situ resources and financed step by step by a public and private partnership can enable the long term development of the region it serves.

The pioneering OASIS concept is technically credible because most of the critical technologies required to develop it have already been studied. The technologies of the first phase (on orbit propellant production, on orbit refueling, autonomous rendezvous and docking, etc.) have reached a technology readiness level of at least 4. Other actors such as United Launch Alliance already have concepts of fuel depots. The biggest unknown resides in ultracold lunar ice extraction which could be answered by NASA’s RESOLVE mission planned for 2017.

\section{Acknowledgments}

This paper is based on the OASIS report (Team OASIS, 2012) which was prepared by an international group of 34 participants from 19 countries during the course of the Space Studies Program (SSP) 2012 at the International Space University (ISU): Melanie Clegg, Vincent Coache, Tyler Dwyer, Shady El Azab, Kristin Freeman, Shai Gerner, Marc Gick, Fei Guan, Yuri Ishizu, Sebastian Klaus, Marc Labriet, Mikkel Ladegaard, Frederico Larangeira, Luliang Lou, Zhuoyan Lu, Qinglang Luo, Nuno Loureiro, Julie Mason, Narasimha Murthy Neeruganti Narasimhulu, Paul Nizenkov, Fredrik Persson, Udrivolf Pica, Lucie Poulet, Maxime Puteaux, Dmitry Rachkin, Eirini Maria Sfantzikaki, Yuan Si, Chrishma Singh-Derewa, Duarte Sousa, Rui Sousa, Suki Dauda Sule, Anna Szwemin, Graeme Taylor, and Aliya Valiyff.

We would like to thank the faculty, staff and alumni of the International Space University for the tremendous support provided at the Florida Institute of Technology, Melbourne, Florida in the Space Studies Program 2012 held during from June - August of 2012. In addition the authors would like to thank NASA, Kennedy Space Center for supporting this effort and the ISU SSP. 


\section{References}

${ }^{1}$ Clegg, et. al., “Operations and Service Infrastructure for Space. Team Project: Spaceports,” Final Report Edition, SSP12, Strasbourg, France, International Space University (ISU), 2012.

${ }^{2}$ Larson, W., Gill, T., Mueller, R.P., and Brink, J., “Establishment of a Spaceport Network Architecture,” Proc. 63 ${ }^{\text {rd }}$

International Astronautics Conference, Paper no. IAC-12-D3.2.6, Naples, Italy, 2012.

${ }^{3}$ Eckart, P., "The Lunar Base Handbook, Second Edition,” Space Technology Series, 2006.

${ }^{4}$ Blair, B. R., Diaz, J., Duke, M. B., Lamassoure, E., Easter R., Oderman, M., Vaucher, M.“'Space Resource Economic Analysis Toolkit: The Case for Commercial Lunar Ice Mining,” Final Report to the NASA Exploration Team, SRD Case 1.0, Center for the Commercial Applications of Combustion in Space (CSM)/ Jet Propulsion Laboratory (JPL)/ CSP Associates, 2002.

${ }^{5}$ Mazarico, E., Neumann, G.A., Smith, D.E., Zuber, M.T., Torrence, M.H.; "Illumination conditions of the lunar polar regions using LOLA topography”; Icarus 211, pp. 1066-1081, 2011.

${ }^{6}$ ESA, “Building a Lunar base with 3D printing”, ESA int, Paris, France, 2013.

${ }^{7}$ Mars-X Team, “Mars X. Team Project,” Final Report Edition, MSc. Program 2013, Strasbourg, France, International Space University (ISU), 2013.

${ }^{8}$ Hopkins, J. and Pratt, W., "Comparison of Deimos and Phobos as Destinations for Human Exploration, and Identification of Preferred Landing Sites,” AIAA Space 2011 Conference, 27 September, 2011.

American Institute of Aeronautics and Astronautics 